\title{
A SCHEME FOR OPTICAL PULSE GENERATION USING OPTOELECTRONIC PHASE LOCKED LOOPS
}

\author{
Madhumita Bhattacharya ${ }^{1}$ \\ ${ }^{I}$ Assistant Professor of Physics, Department of Physics, Guskara Mahavidyalaya, West Bengal
}

\begin{abstract}
A new scheme of optical pulse generation using multiple optoelectronic phase locked loops is proposed. The principle of the scheme is combining three or more coherent optical waves having equal frequency separation. The coherencies of the optical waves are attained by the use of optoelectronic phase locked loops. The repetition frequency of the generated optical pulse is equal to the frequency of the reference microwavel millimeter wave signal source used in the phase locked loop. The intensity profile of the generated optical pulse and the pulse width are calculated. If four coherent optical carriers are combined, the generated optical pulse has a typical width of 2.4 picoseconds. In the proposed scheme the central frequency of the optical pulse and the repetition frequency can be varied. Since in this scheme, the superimposed optical waves are phase locked the associated phase noise of the optical pulse will be low.
\end{abstract}

Keywords: optical pulse generation, optoelectronic phase locked loop, pulse repetition frequency, laser diode, photodiode

\section{INTRODUCTION}

Generation of high repetition rate ultra short optical pulse is an important topic of research due to its application in high speed optical time division multiplexed (OTDM) communication system and in optical signal processing [1-10]. To exploit the terahertz bandwidth of optical fiber around $1.55 \mu \mathrm{m}$, different multiplexing techniques are widely used. Wavelength division multiplexing (WDM) and time division mutiplexing OTDM communication system has the capability of carrying a high data rate of $100 \mathrm{~Gb} / \mathrm{s}$ over a single optical channel. It also finds potential application as optical clocks required in optical computing. Conventional methods of optical pulse generation consists of gain switching, Q switching and mode locking of semiconductor lasers. The optical pulse generated from gain switching and Q switching of laser diodes suffer from phase fluctuation. Mode locked lasers generate optical pulse with repetition rate limited to $40 \mathrm{Ghz}$.

In this paper, we propose a new method of pulse generation having short pulse width and high repetition frequency by combining a number of coherent light waves. The coherency of the optical waves are attained by the phase locked loops. In this scheme, as the output lightwaves of the laser diodes are phase locked, the phase noise of the optical waves are greatly reduced. The phase noise of the generated optical pulse will also be low.

\subsection{System Description}

The schematic circuit diagram of the proposed optical pulse generator is shown in Fig.1. It consists of multiple optoelectronic phases locked loops (OEPLL). A single
OEPLL consists of two laser diodes (LD1 and LD2), a photodiode, a reference microwave signal source, a electronic mixer and a low pass filter. The laser diodes have free running frequencies $f_{1}$ and $f_{2}$ respectively. The frequency difference between the laser diodes is maintained at a constant value, which is equal to that of the microwave reference signal frequency. Considering a single OEPLL, lightwaves from the laser diodes LD1 and LD2 are heterodyned in a wideband photodiode (PD1). At the output of the photodiode we get an electrical signal having frequency $\left(f_{2}-f_{1}\right)$. The free running frequencies of the laser diodes are so chosen that their difference is almost equal to that of the reference microwave signal. The signal having frequency $\left(f_{2}-f_{1}\right)$ and the output of the reference oscillator are mixed in the electronic mixer. The signal at the output of the RF mixer is low pass filtered and is added to the bias of the laser diode LD2. In the phase locked condition, the lightwaves from the laser diodes LD1 and LD2 are made coherent and the frequency difference ( $\left.f_{2}-f_{1}\right)$ is exactly equal to that of the reference microwave source $\left(f_{r}\right)$. By using the second OEPLL, the lightwaves from LD2 and LD3 are phase locked and the frequency difference $\left(f_{3}-f_{2}\right)$ is maintained constant. The output of the laser diode LD2 enters the second OEPLL through the half mirror (HM2). Thus, by using two OEPLLs, we get three coherent lightwaves having constant frequency differences. These three lightwaves are directed into the optical combiner and superimposed to generate the optical pulse. The repetition frequency of the pulse is equal to the frequency of the reference microwave source. Similarly, the lightwaves from 
the laser diodes LD3 and LD4 (not shown in Figure 1) can be made coherent by using the third OEPLL. As the number of OEPLLs is increased, the number of coherent lightwaves also increases. These multiple lightwaves are combined to generate the optical pulse.

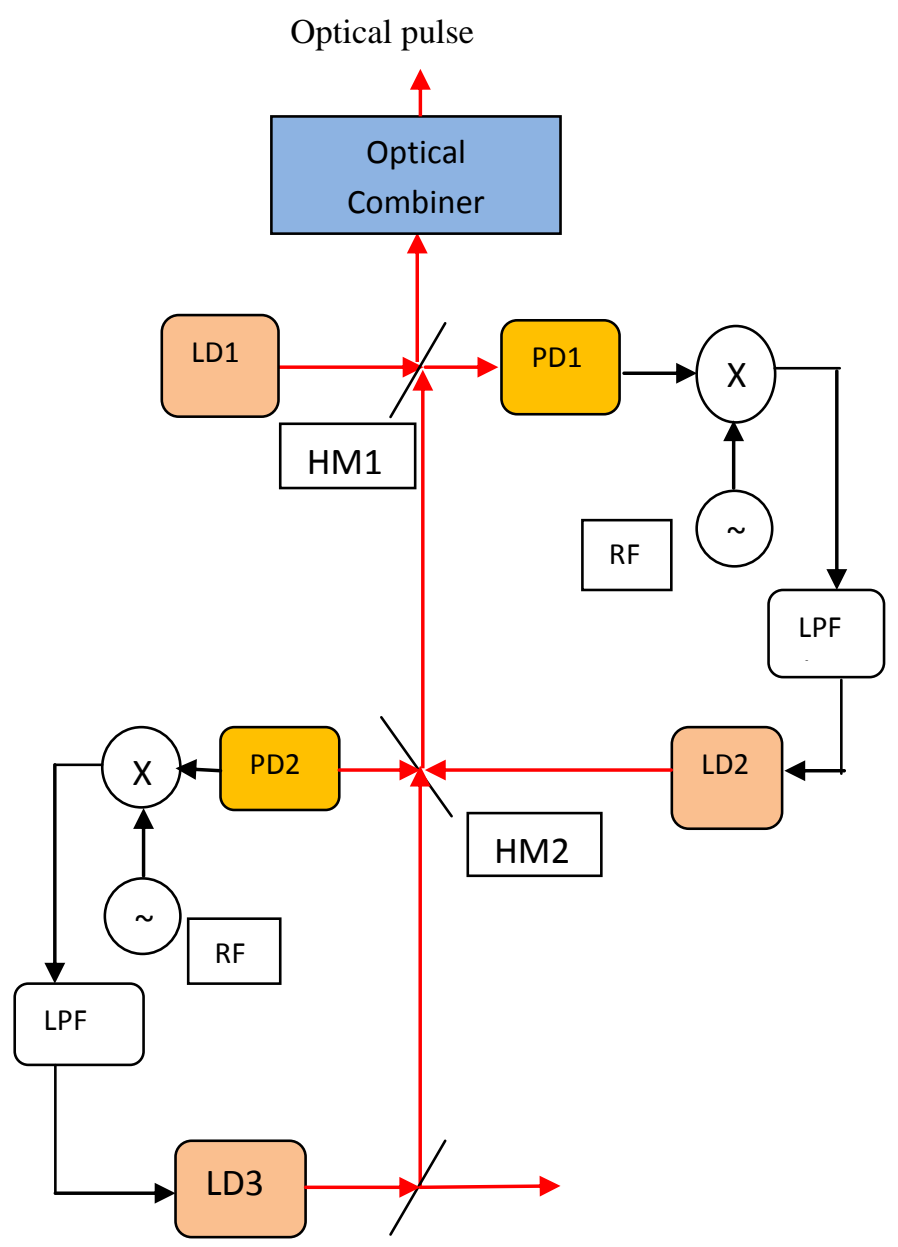

Fig.1: A schematic circuit diagram of the proposed optical pulse generator. LD: laser diodes; PD: photodiodes; X: electronic mixer; RF: reference microwave signal source; LPF : low pass filter; HM: half mirror ;

Electrical path:

Optical path:

\section{ANALYSIS}

In Fig.1, there are two OEPLLs which are assumed to be under locked condition. Let the electric fields of the output lightwaves from LD1, LD2 and LD3 be represented as

$$
\begin{aligned}
& a_{2}=\left|a_{2}\right| \exp \left[j\left(\omega_{2}+\phi_{2}\right)\right] \\
& \text { and } a_{3}=\left|a_{3}\right| \exp \left[j\left(\omega_{3}+\phi_{3}\right)\right]
\end{aligned}
$$

respectively.

As the OEPLLs are balanced, the angular frequencies satisfies the condition

$$
\left(\omega_{3}-\omega_{2}\right)=\left(\omega_{2}-\omega_{1}\right)=\omega_{r}
$$

Where $\omega_{r}$ is the angular frequency of the reference microwave signal source. The reference microwave signal used in the OEPLLs can be obtained from a single reference source. The reference source can be a Gunn oscillator having a frequency of $60 \mathrm{GHz}$.

Using the scattering matrix of the half mirror [11], the composite lightwaves that are combined can be written as

$$
\begin{aligned}
a_{o}(t)= & j\left[\frac{\left|a_{1}\right|}{\sqrt{2}} \exp j\left(\omega_{1} t+\phi_{1}\right)+\frac{\left|a_{2}\right|}{2} \exp j\left(\omega_{2}+\phi_{2}\right)\right. \\
& \left.+\frac{\left|a_{3}\right|}{2 \sqrt{2}} \exp j\left(\omega_{3} t+\phi_{3}\right)\right]
\end{aligned}
$$

These three coherent optical waves are superimposed to generate the optical pulse. Let us consider the output optical powers of the lasegdiodes are identical. $\left|a_{1}\right|=\left|a_{2}=\right| a_{3}|=| a \mid$ . So we can write eqn.(5) as

$$
\begin{aligned}
a_{o}(t)= & j \frac{\left|a_{1}\right|}{\sqrt{2}}\left[\exp j\left(\omega_{1} t+\phi_{1}\right)+\frac{1}{\sqrt{2}} \exp j\left(\omega_{2}+\phi_{2}\right)\right. \\
& \left.+\frac{1}{2} \exp j\left(\omega_{3} t+\phi_{3}\right)\right]
\end{aligned}
$$

The normalized intensity of the optical pulse is calculated to be

$$
\begin{aligned}
I_{N}(t)= & {\left[7+4 \sqrt{2} \cos \left(\omega_{r} t+\phi_{2}-\phi_{1}\right)+\right.} \\
& 2 \sqrt{2} \cos \left(\omega_{r} t+\phi_{3}-\phi_{2}\right) \\
& \left.+4 \cos \left(2 \omega_{r} t+\phi_{3}-\phi_{1}\right)\right]
\end{aligned}
$$

$$
a_{1}=\left|a_{1}\right| \exp \left[j\left(\omega_{1}+\phi_{1}\right)\right]
$$


The repetition frequency of the optical pulse is equal to the frequency of the applied reference microwave signal source. If a Gunn oscillator of frequency $60 \mathrm{GHz}$ is used as the reference source the repetition frequency is also $60 \mathrm{GHz}$. In Fig.2, the normalized intensity of the generated optical pulse is shown as a function of time, considering two OEPLLs. The two optical pulse corresponds to repetition frequencies of $60 \mathrm{GHz}$ and $100 \mathrm{GHz}$ respectively. Fig. 3 shows the intensity of a single pulse. The optical pulse widths are calculated to be $5.2 \mathrm{ps}$ and 3.2 ps for the two cases. In numerical calculations we have assumed $\phi_{1}=\phi_{2}=\phi_{3}=0$.

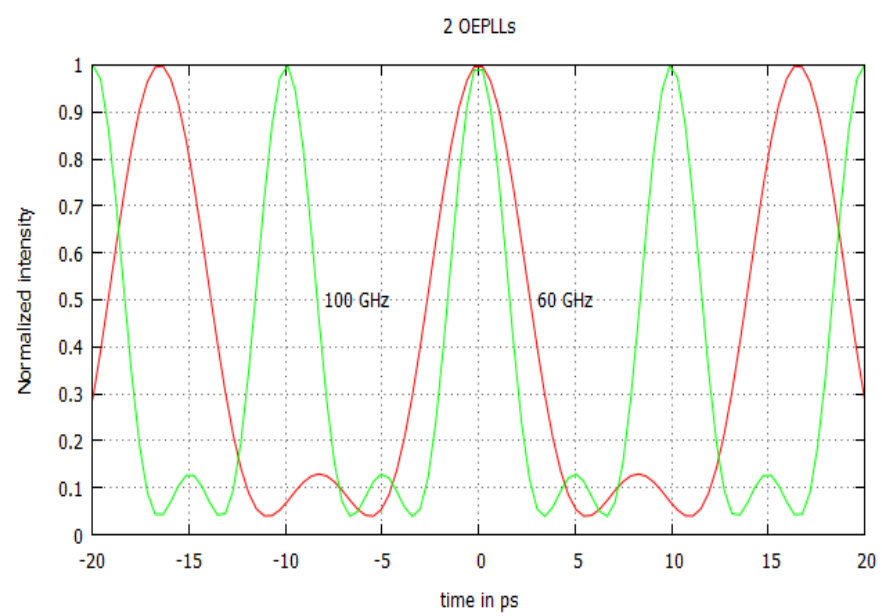

Fig.2. Variation of the normalized intensity of the generated optical pulse as a function of time using repetition frequency as a parameter

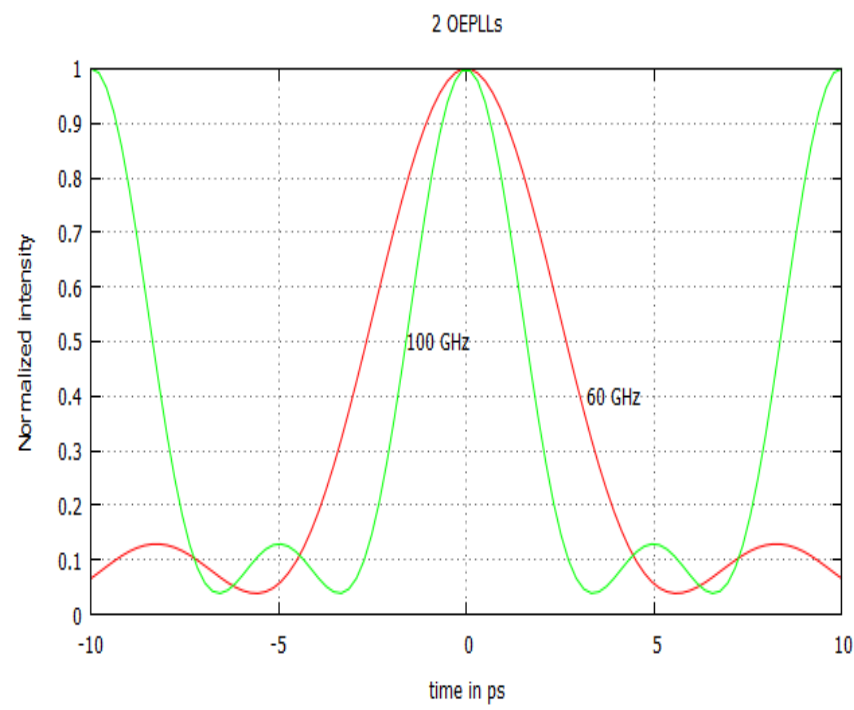

Fig.3. Same as in Fig.2 for a single pulse.
If we consider 3 OEPLLs, at the input of the optical combiner there are 4 optical coherent waves. The 4 laser diodes used have frequencies $\omega_{1}, \omega_{2}, \omega_{3}$ and $\omega_{4}$ respectively. They satisfy the condition

$\left(\omega_{4}-\omega_{3}\right)=\left(\omega_{3}-\omega_{2}\right)=\left(\omega_{2}-\omega_{1}\right)=\omega_{r}$.

In this case the normalized intensity is calculated to be

$$
\begin{aligned}
& I_{N}(t)=\left[15+8 \sqrt{2} \cos \left(\omega_{r} t+\phi_{2}-\phi_{1}\right)+\right. \\
& 4 \sqrt{2} \cos \left(\omega_{r} t+\phi_{3}-\phi_{2}\right)+2 \sqrt{2} \cos \left(\omega_{r} t+\phi_{4}-\phi_{3}\right) \\
& +8 \cos \left(2 \omega_{r} t+\phi_{3}-\phi_{1}\right)+4\left(2 \omega_{r} t+\phi_{4}-\phi_{2}\right)+ \\
& \left.4 \sqrt{2} \cos \left(3 \omega_{r} t+\phi_{4}-\phi_{1}\right)\right]
\end{aligned}
$$

The variation of the normalized intensity of the generated pulse as a function of time is shown in Fig.4. From Fig.5, we can notice as the number of coherent light wave are increased the optical pulse width decreases. Again, the pulse width also decreases for higher repetition rate pulse. The calculated pulse width is found to be 4 ps and 2.4 ps respectively.

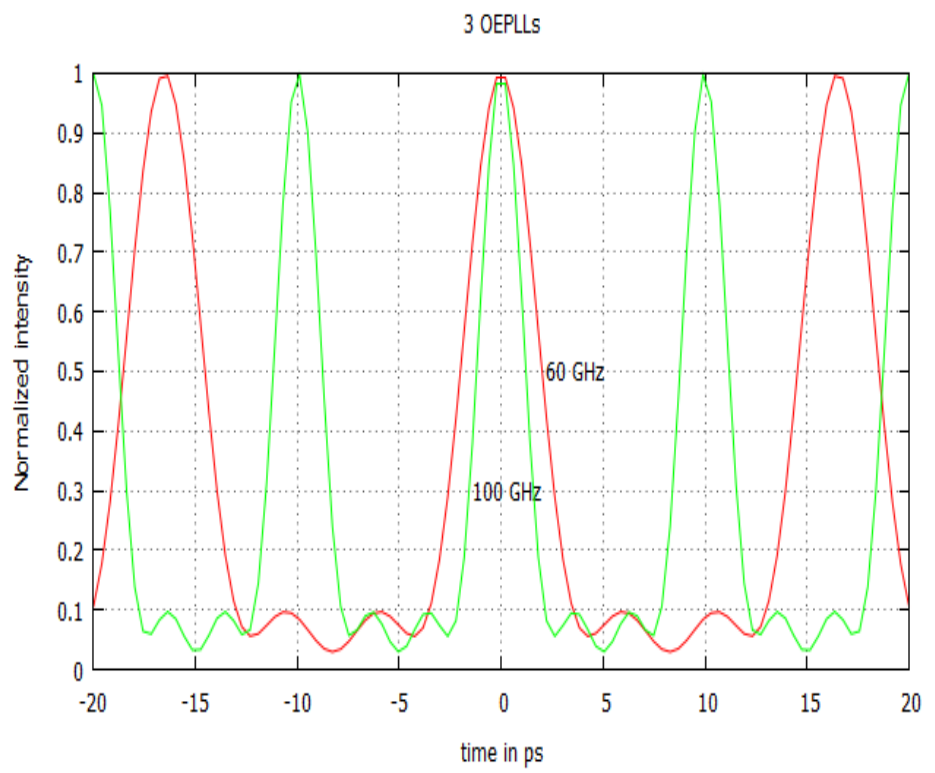

Fig.4. Intensity profile of the optical pulse when 3 OEPLLs are used taking repetition frequency as a parameter 


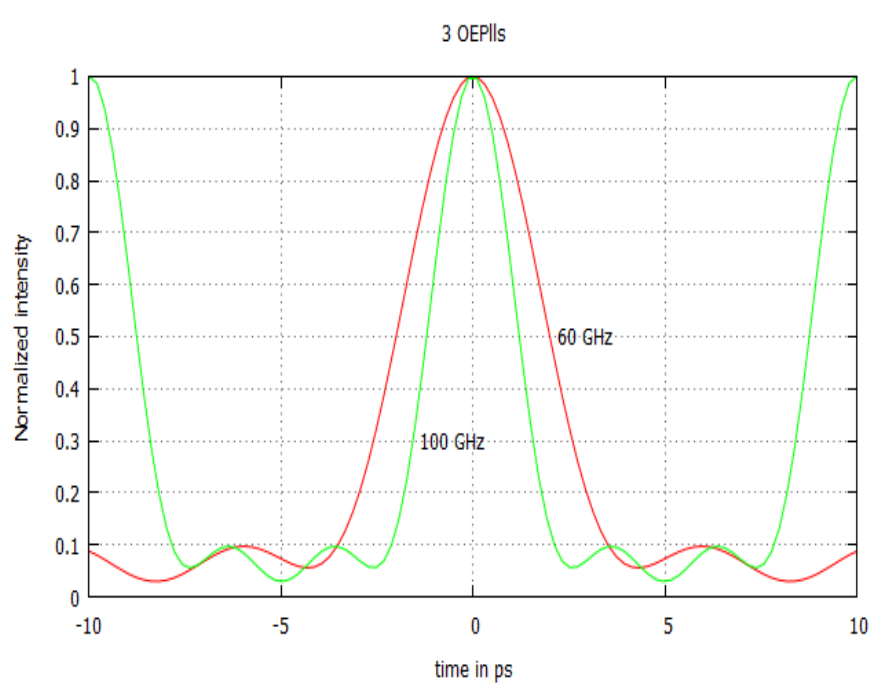

Fig.5. Same as in Fig.4 for a single pulse

The calculated optical pulse widths for the different cases are shown in Table-1.

Table-1

\begin{tabular}{|c|c|c|}
\hline $\begin{array}{c}\text { No. of } \\
\text { OEPLLs }\end{array}$ & $\begin{array}{c}\text { Optical pulse } \\
\text { repetition frequency }\end{array}$ & $\begin{array}{c}\text { Optical } \\
\text { Pulse width }\end{array}$ \\
\hline 1 & $60 \mathrm{GHz}$ & $5.2 \mathrm{ps}$ \\
\hline 1 & $100 \mathrm{GHz}$ & $3.2 \mathrm{ps}$ \\
\hline 2 & $60 \mathrm{GHz}$ & $4 \mathrm{ps}$ \\
\hline 2 & $100 \mathrm{GHz}$ & $2.4 \mathrm{ps}$ \\
\hline
\end{tabular}

\section{CONCLUSIONS}

A new method of optical pulse generation using optoelectronic phase locked loops is proposed in this paper. Three coherent continuous lightwaves are combined to generate the optical pulse. The coherencies of the lightwaves are maintained by the use of optoelectronic phase locked loops. The repetition frequency of the generated optical pulse is equal to the frequency of the reference microwave signal source. The intensity profile of the generated optical pulse is calculated.

\section{REFERENCES}

[1] H. Murata, A. Moromoto, T. Kobayashi, and S. Yamamoto, "Optical pulse generation by electrooptic modulation method and its application to integrated ultrashort pulse generators", IEEE Journal of Selected Topics on Quantum Electronics, vol. 6, pp. 1325-1331, 2000.

[2] J. H. Lee, Y. M. Chang, Y-G, Han, S. H. Kim and S. B. Lee, "2 5 times tunable repetition-rate multiplication of a $10 \mathrm{GHz}$ pulse sources using a linearly tunable, chirped fiber Bragg gratting", Optics Express, no. 17, vol. 12, pp. 3900-3905, 2004.
D. Miyamoto, K. Mandai, T. Kurokawa, S. Takeda, T. Shioda, and H. Tsuda, "Waveform controllable optical pulse generation using an optical pulse synthesizer", IEEE Photonics Technology Letters, vol. 18, no. 5, pp. 721-723, March 2006.

[4] S. Das and T. Chattopadhyay, "A method of tunable high repetition-rate picosecond optical pulse generation using injection locking of laser diodes", Applied Physics B (Laser and Optics), vol-83, pp. 549-551 April 2006.

[5] S. Xiao, L. Hollberg, N.R.Newbury, and S.A. Diddams, "Towards a low jitter $10 \mathrm{GHz}$ pulsed source with an optical frequency comb generator, "Optics Express, vol.16, pp. 8498-8508, 2008.

[6] S. Takasaka, Y.Ozeki, S.Maniki, and M. Sakano, “ External synchronization of $160 \mathrm{GHz}$ optical beat signal by optical phase-locked loop technique" IEEE Photonics Technology Letters, Vol.18, no.23, pp.24572459, 2006.

[7] T. Inoue, J. Hiroishi, T.Yagi, and Y. Mimura, “ Generation of in-phase pulse train from optical beat signal," Optics Letters, vol.32, pp.1596-1598, 2009.

[8] J. Li, B. Kuo, and K. Wong, "Ultra-wideband pulse generation based on cross-gain modulation in fiber optical parametric amplifier," IEEE Photon. Technol. Lett., vol. 21, no. 4, pp. 212-214, Feb. 2009.

[9] H. Huang, K. Xu, J. Q. Li, J.Wu, X. B. Hong, and J. T. Lin, "UWBpulse generation and distribution using NOLM based optical switch,"J. Lightw. Technol., vol. 26, no. 15, pp. 2635-2640, Aug. 1, 2008.

[10] T. Chattopadhyaya and P. Bhattacharya, "A scheme for low noise optical pulse generation, " Journal of Optics, vol.42, no. 2, pp.148-155, 2013.

[11] M. Bhattacharya and T. Chattopadhyay, "An optical limiter-discriminator using synchronized laser diodes, " 1999 J. Opt. A: Pure Appl. Opt. Volume 1, Number 5, 1999 , pp. 626-628. 